\title{
Improving The Performance of Linear Systems by Adding a Hybrid Loop: The Output Feedback Case
}

\author{
Francesco Fichera, Christophe Prieur, Sophie Tarbouriech and Luca Zaccarian
}

\begin{abstract}
In this paper, we extend the state feedback results of Prieur et al. (NOLCOS 2010) to the linear output feedback case, when the plant state is not available and is estimated via a Luenberger observer. Two techniques, based on different reset maps and flow and jump sets and guaranteeing global practical asymptotic stability of the origin of the closed-loop system, are proposed. The effectiveness of the solutions is illustrated on a simulation example where we show suitable reduction of the output overshoot.
\end{abstract}

Index Terms-Hybrid systems, practical stability, reset controllers.

\section{INTRODUCTION}

In an attempt to enlarge the frontiers of control theory, hybrid systems have proved to be capable to remove some fundamental limitations of classical theory. New mathematical tools that go beyond classical analysis have been developed to handle this class of dynamical systems having both continuous and discrete behavior, [1]-[3]. Hybrid systems are useful, for example, to robustly stabilize nonlinear systems not stabilizable by smooth feedback, [4]-[6]. Furthermore, first order reset elements (FORE) or reset systems have been shown to overcome some intrinsic limitations of linear control systems, see [7]-[9] and references therein. Finally, suitably resetting (not necessarily in zero, as in the above reset systems works) a controller state upon certain conditions can, in general, lead to more desirable closed-loop behavior, see for example the optimal reset law proposed in [10] for switching systems. Hybrid controllers have been also addressed in the context of the so-called impulsive systems literature [11], [12], where the discrete jumps of the state are described by way of impulses suitably acting on the system.

Recently in [13], [14], we proposed a technique to design hybrid control loops whose jumps are triggered by suitable Lyapunov-like conditions (such as the non-increase of some Lyapunov-like functions of the closed-loop state). The potential of that technique was illustrated, for example, by an effective method for reducing the overshoot of a suitable output. However, the approach in [13], [14] heavily relies on the knowledge of the plant state which is not only used

Work supported by HYCON2 Network of Excellence "Highly-Complex and Networked Control Systems", grant agreement 257462.

F. Fichera, S. Tarbouriech and L. Zaccarian are with CNRS, LAAS, 7 avenue du colonel Roche, F-31400 Toulouse, France and Univ. de Toulouse, LAAS, F-31400 Toulouse, France. ffichera@laas.fr, tarbourelaas.fr, lzaccarielaas.fr

C. Prieur is with Department of Automatic Control, Gipsa-lab, 961 rue de la Houille Blanche, BP 46, 38402 Grenoble Cedex, France, christophe.prieurdgipsa-lab.grenoble-inp.fr to decide the optimal reset value (as in [10]), but also to detect whether the state is in the jump or flow set (namely, decide whether or not to reset the controller).

In this paper, we extend the work of [13], [14] by removing the heavy assumption that the plant state is available. To this aim, similar to [10], we introduce a Luenberger observer in the closed loop and rely on the arising plant state estimate for the design of flow and jump sets and reset maps, which asymptotically recover those of our previous work as the estimation error converges to zero.

Our approach shares some similarities with [10]. However, while [10] focuses on the design of the switching law (or reset map), here we also design the flow and jump sets based on the estimated plant state.

Since the plant state is not accessible, we cannot reuse the same proof technique of [13], [14], where the knowledge of the whole state allowed to prevent increase of the Lyapunov function by triggering suitable resets in certain regions. Here, in the output feedback case, we are constrained to only define jump and flow sets based on a partial state information and then prove stability properties using additional Lyapunov arguments. We propose two schemes, one of them using similar sets to those of [13], [14] redefined on the observer state, the other one exploiting an additional term driven by the output observation error for the definition of both flow and jump sets and the reset map. All of our results here establish practical asymptotic stability of the closed loop because we forbid jumps in an arbitrarily small ball around the origin to avoid defective Zeno solutions that prevent the observation error from converging to zero (a similar approach has also been used in [15]).

The rest of the paper is organized as follows. Some notation and basic definitions on hybrid systems are given next. In Section II, the main results are presented, a subsection for each control schemes is introduced with a further subsection with some comparative remarks. Some simulations and comparisons are presented in Section III.

Notation and preliminaries. Given a vector $x, x^{T}$ denotes the transpose of $x$. The scalar product is denoted by $\langle\cdot, \cdot\rangle$ and the Euclidean norm by $|\cdot| . \mathbb{R}$ denotes the set of real numbers, $\mathbb{R}_{\geq 0}$ denotes the set of non-negative real numbers and $\mathbb{N}$ the one of non-negative integers. For a matrix $M$, $\mathrm{He}(M)=M+M^{T}$ and for any symmetric matrix, * stands for a symmetric term. For a positive integer $n, I_{n}$ (respectively, $0_{n}$ ) denotes the identity matrix (respectively, 
the null matrix) in $\mathbb{R}^{n \times n}$. $0_{n, m}$ with $n \neq m$ and $n, m$ positive integers, denotes the null matrix in $\mathbb{R}^{n \times m}$. The subscripts may be omitted when there is no ambiguity. Given a compact set $\mathcal{A}$, the notation $|x|_{\mathcal{A}}=\min \{|x-y|: y \in \mathcal{A}\}$ indicates the distance of the vector $x$ from the set $\mathcal{A}$. The boundary of $\mathcal{A}$ is denoted $\partial \mathcal{A}$. If $\mathcal{A}$ is the origin then $|x|_{\mathcal{A}}=|x|$.

For an introduction of the hybrid systems framework, see [2]. A hybrid system is represented as

$$
\left\{\begin{array}{r}
\dot{x}=f(x) \text { if } x \in C \\
x^{+}=g(x) \text { if } x \in D
\end{array}\right.
$$

where $x \in \mathbb{R}^{n}$ is the state space of the hybrid system, $C \subseteq$ $\mathbb{R}^{n}$ is the flow set, $D \subseteq \mathbb{R}^{n}$ is the jump set, while $f: C \rightarrow$ $\mathbb{R}^{n}$ and $g: D \rightarrow \mathbb{R}^{n}$ are single-valued mappings, called the flow map and the jump map, respectively. A solution $x$ consists in a function $x: \operatorname{dom}(x) \rightarrow \mathbb{R}^{n}$, where $\operatorname{dom}(x)$ is the hybrid time domain, defined as a suitable set of $\mathbb{R}_{\geq 0} \times \mathbb{N}$.

According to [2], we introduce next only the definition that will be considered in the rest of the paper. For further details see also [3], [16]-[18]. A solution of (1) is said to be complete if $\operatorname{dom}(x)$ is unbounded and Zeno if it is complete but the projection of $\operatorname{dom}(x)$ onto $\mathbb{R}_{\geq 0}$ is bounded.

Definition 1: Given the hybrid system (1), a compact set $\mathcal{A} \subset \mathbb{R}^{n}$ is said to be

- stable for (1): if for each $\epsilon>0$ there exists $\delta>0$ such that each solution $x$ to (1) with $|x(0,0)|_{\mathcal{A}} \leq \delta$ satisfies $|x(t, j)|_{\mathcal{A}} \leq \epsilon$ for all $(t, j) \in \operatorname{dom}(x)$;

- attractive for (1): if every solution $x$ to (1) is complete and satisfies $\lim _{t+j \rightarrow \infty}|x(t, j)|_{\mathcal{A}}=0$;

- globally asymptotically stable (GAS) for (1): if it is both stable and attractive for (1).

\section{MAIN RESULTS}

\section{A. Controller architecture}

In this paper we consider a LTI plant $\mathcal{P}$, represented by

$$
\mathcal{P}\left\{\begin{array}{l}
\dot{x}_{p}=\bar{A}_{p} x_{p}+\bar{B}_{p} u \\
y_{p}=\bar{C}_{p} x_{p}+\bar{D}_{p} u
\end{array}\right.
$$

with $x_{p} \in \mathbb{R}^{n_{p}}, u \in \mathbb{R}^{p}$ and $y_{p} \in \mathbb{R}^{q}$ and satisfying the following assumption.

Assumption 1: The pair $\left(\bar{C}_{p}, \bar{A}_{p}\right)$ is detectable.

$\circ$

Now, let us introduce the vectors $\hat{x}_{p} \in \mathbb{R}^{n_{p}}$ and $x_{c} \in \mathbb{R}^{n_{c}}$, which combined together will give us our controllers state $\bar{x}=\left[\begin{array}{ll}\hat{x}_{p}^{T} & x_{c}^{T}\end{array}\right]^{T}$. As Figure 1 shows, the hybrid controller architecture, we propose, can be represented as: a continuoustime controller with state $x_{c}$, experiencing jumps and governing the closed-loop dynamics during flows; a continuoustime Luenberger observer with state $\hat{x}_{p}$, used for the jump dynamics only; and a supervisor, which accomplishes three tasks:

- receives the signals $x_{c}, \hat{x}_{p}$ (and $y_{p}, \hat{y}_{p}$ for the solution in Section II-C);
- evaluates if the controller state is in the flow or jump sets;

- resets the state $x_{c}$ if the controller state is in the jump set.

Here we propose two hybrid controllers differing only in the supervisor block (namely in the jump map, in the flow and jump sets and in the information received by the supervisor). For the first technique, described in Section II$\mathrm{B}$, the supervisor receives only the signals represented by solid arrows (see Fig. 1), whereas in the second technique, described in Section II-C, the supervisor receives also the output estimate from the observer, $\hat{y}_{p}$, and the plant output, $y_{p}$ (dashed arrows).

The continuous dynamics of both of our controllers and the plant input $u$ satisfy the same equations given next

$$
\begin{aligned}
{\left[\begin{array}{c}
\dot{\hat{x}}_{p} \\
\dot{x}_{c}
\end{array}\right] } & =\left[\begin{array}{cc}
A_{e} & B_{e} \bar{C}_{c} \\
0 & \bar{A}_{c}
\end{array}\right]\left[\begin{array}{l}
\hat{x}_{p} \\
x_{c}
\end{array}\right]+\left[\begin{array}{c}
B_{e} \bar{D}_{c}+L \\
\bar{B}_{c}
\end{array}\right] y_{p} \\
& :=A_{\mathcal{H}} \bar{x}+B_{\mathcal{H}} y_{p} \\
u & =\left[\begin{array}{ll}
0 & \bar{C}_{c}
\end{array}\right]\left[\begin{array}{l}
\hat{x}_{p} \\
x_{c}
\end{array}\right]+\bar{D}_{c} y_{p} \\
& :=C_{\mathcal{H}} \bar{x}+D_{\mathcal{H}} y_{p}
\end{aligned}
$$

where $A_{e}=\bar{A}_{p}-L \bar{C}_{p}, B_{e}=\bar{B}_{p}-L \bar{D}_{p}$ and all the other matrices are design parameters that will be defined later.

Assumption 2: The interconnection (2),(3) is well-posed, that is, the matrix $\left(I-\bar{D}_{p} \bar{D}_{c}\right)$ is non-singular.

Defining the closed-loop state $x=\left[\hat{x}_{p}^{T} x_{c}^{T} e^{T}\right]^{T}$, we can rewrite (2),(3) as

$$
\begin{aligned}
{\left[\begin{array}{c}
\dot{\hat{x}}_{p} \\
\dot{x}_{c} \\
\dot{e}
\end{array}\right] } & =\left[\begin{array}{ccc}
A_{p} & B_{p} & B_{o} \\
B_{c} & A_{c} & B_{c} \\
0 & 0 & A_{e}
\end{array}\right]\left[\begin{array}{c}
\hat{x}_{p} \\
x_{c} \\
e
\end{array}\right]:=A x \\
y_{p} & =\left[\begin{array}{lll}
C_{p} & C_{c} & C_{p}
\end{array}\right] x:=C x,
\end{aligned}
$$

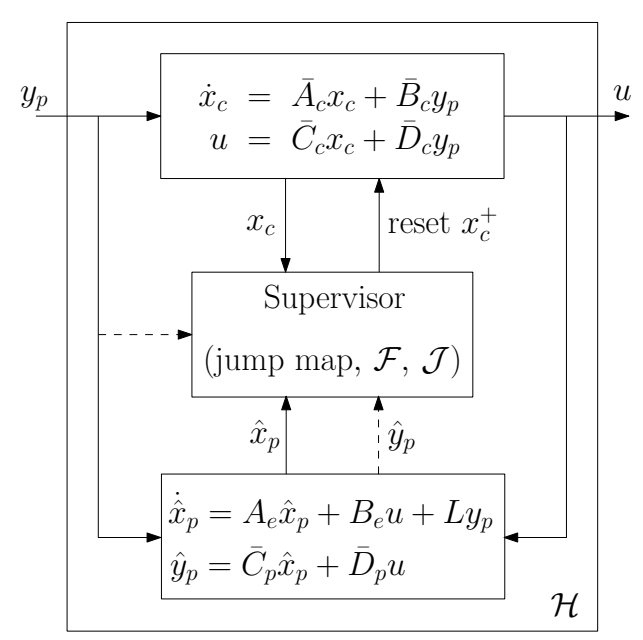

Fig. 1. Hybrid controllers architecture. 
where

$$
\begin{gathered}
{\left[\begin{array}{c|c|c}
A_{p} & B_{p} & B_{o} \\
\hline B_{c} & A_{c} & B_{c} \\
\hline C_{p} & C_{c} & C_{p}
\end{array}\right]=\left[\begin{array}{c|c|c}
\bar{A}_{p} & \bar{B}_{p} \bar{C}_{c} & L \bar{C}_{p} \\
\hline 0 & A_{c} & 0 \\
\hline 0 & 0 & 0
\end{array}\right]} \\
+\left[\begin{array}{c}
\frac{\bar{B}_{p} \bar{D}_{c}}{\bar{B}_{c}} \\
\hline I
\end{array}\right] X\left[\bar{C}_{p}\left|\bar{D}_{p} \bar{C}_{c}\right| \bar{C}_{p}\right],
\end{gathered}
$$

with $X=\left(I-\bar{D}_{p} \bar{D}_{c}\right)^{-1}$ well defined from Assumption 2 and $A_{e}$ defined after (3).

Remark 1: The gain $L \in \mathbb{R}^{n_{p} \times q}$ is selected such a way that the matrix $A_{e}$ is Hurwitz (see Assumption 1). Defining $e=x_{p}-\hat{x}_{p}$ and from (4a), we have $\dot{e}=\dot{x}_{p}-\dot{\hat{x}}_{p}=$ $A_{e} e$. This proves that the controller sub-state $\hat{x}_{p}$ converges exponentially to the plant state $x_{p}$, during flow.

Remark 2: The plant input $u$ depends only on $x_{c}$ and $y_{p}$ (see (3b)), this means that the information coming from the observer is used only by the supervisor (see Fig. 1). This peculiar architecture was also used in [10].

In the sequel, we provide two designs of the supervisor block of Fig. 1, both of them generalizing the result of [13, Section 3] (see also [14]) to the output feedback case.

\section{B. Reset rule replacing $x_{p}$ with $\hat{x}_{p}$}

Once the observer in the lowest block of Fig. 1 is in place, the most natural approach to generalize [13, Section $3]$ is to replace the plant state $x_{p}$ by its estimate $\hat{x}_{p}$ in all the relations present in the supervisor block. We pursue this approach here and establish its effectiveness. The hybrid controller design relies on the following assumption which is a simplified version of [13, Assumption 1] for the linear case. Note that the corresponding inequality can be manipulated and transformed into a convex one by following the approach in [13, Section 5], see also Section III.

Assumption 3: Given the plant $\mathcal{P}$, there exist a symmetric matrix $P=\left[\begin{array}{cc}P_{p} & P_{p c} \\ P_{p c}^{T} & P_{c}\end{array}\right]>0$ and a scalar $\alpha>0$ such that

$$
\begin{gathered}
\operatorname{He}\left(\left(P_{p}-P_{p c} P_{c}^{-1} P_{p c}^{T}\right)\left(A_{p}-B_{p} P_{c}^{-1} P_{p c}^{T}\right)\right) \\
<-\alpha\left(P_{p}-P_{p c} P_{c}^{-1} P_{p c}^{T}\right) .
\end{gathered}
$$

We select the hybrid controller of Fig. 1 as

$$
\begin{array}{rlrl}
\dot{\bar{x}} & =A_{\mathcal{H}} \bar{x}+B_{\mathcal{H}} y_{p} & \text { if } \bar{x} \in \mathcal{F} \\
{\left[\begin{array}{l}
\hat{x}_{p}^{+} \\
x_{c}^{+}
\end{array}\right]} & =\left[\begin{array}{cr}
I & 0 \\
K_{p} & 0
\end{array}\right]\left[\begin{array}{l}
\hat{x}_{p} \\
x_{c}
\end{array}\right] \quad \text { if } \bar{x} \in \mathcal{J} \\
u & =C_{\mathcal{H}} \bar{x}+D_{\mathcal{H}} y_{p}
\end{array}
$$

with the following selections for the flow and jump sets

$$
\begin{aligned}
\mathcal{F} & =\left\{\bar{x}: \bar{x}^{T} N \bar{x} \leq-\tilde{\alpha} \bar{x}^{T} P \bar{x} \text { or } \bar{x}^{T} P \bar{x} \leq \rho\right\} \\
\mathcal{J} & =\left\{\bar{x}: \bar{x}^{T} N \bar{x} \geq-\tilde{\alpha} \bar{x}^{T} P \bar{x} \text { and } \bar{x}^{T} P \bar{x} \geq \rho\right\}
\end{aligned}
$$

where

$$
N=\operatorname{He}\left(P\left[\begin{array}{ll}
A_{p} & B_{p} \\
B_{c} & A_{c}
\end{array}\right]\right)
$$

and $0<\tilde{\alpha} \leq \alpha$ and the scalar $\rho>0$ is related to the practical stability result established next and can be chosen arbitrarily small.

Using (4), the interconnection (2),(6) becomes

$$
\begin{array}{rlrl}
\dot{x} & =A x & \text { if } x \in\left(\mathcal{F} \times \mathbb{R}^{n_{p}}\right) \\
{\left[\begin{array}{c}
\hat{x}_{p}^{+} \\
x_{c}^{+} \\
e^{+}
\end{array}\right]} & =\left[\begin{array}{ccc}
I & 0 & 0 \\
K_{p} & 0 & 0 \\
0 & 0 & I
\end{array}\right]\left[\begin{array}{c}
\hat{x}_{p} \\
x_{c} \\
e
\end{array}\right] \quad \text { if } x \in\left(\mathcal{J} \times \mathbb{R}^{n_{p}}\right) \\
y_{p} & =C x &
\end{array}
$$

which corresponds to our hybrid closed-loop system.

Theorem 1: Consider a plant-controller pair (2), (6) with the sets $\mathcal{F}$ and $\mathcal{J}$ in $(7), K_{p}=-P_{c}^{-1} P_{p c}^{T}$, satisfying Assumptions 1, 2 and 3 and with $A_{e}$ Hurwitz.

Then the hybrid controller (6) guarantees that the set

$$
\mathcal{A}:=\{\bar{x}: V(\bar{x}) \leq \rho\} \times\{0\}
$$

where $V(\bar{x})=\bar{x}^{T} P \bar{x}$, is globally asymptotically stable for the closed-loop system (8), namely the origin is practically GAS ( $\rho$ can be arbitrarily small).

Remark 3: Note that whenever $e=0$, so that $x_{p}=\hat{x}_{p}$, the closed-loop (8) coincides with the hybrid closed loop in [13, Proposition 1]. Therefore one should expect the same responses after the observer transient has expired. Nevertheless, a deterioration of the response should be expected, in general, due to the convergence transient of the observer. See Section III for an example showing this behavior.

\section{Enhanced reset rule exploiting $y_{p}-\hat{y}_{p}$}

The solution presented in the previous section succeeds at extending the schemes of [13], [14] to the output feedback case, but it does not directly exploit the instantaneous knowledge of the output error $y_{p}-\hat{y}_{p}$ for the selection of the flow and jump sets and the reset rule. We explore this additional potential here and propose an enhanced scheme which is expected to behave better during the observer transient. The scheme relies once again on the matrices introduced in Assumption 3, plus an extra gain matrix $K_{y} \in \mathbb{R}^{n_{c} \times q}$, which is an additional free tuning parameter. Then, defining $\eta:=y_{p}-\hat{y}_{p}=\bar{C}_{p}\left(x_{p}-\hat{x}_{p}\right)=\bar{C}_{p} e$ and $\zeta=(\bar{x}, \eta)$, the hybrid controller of Fig. 1 is selected as

$$
\begin{array}{rlrl}
\dot{\bar{x}} & =A_{\mathcal{H}} \bar{x}+B_{\mathcal{H}} y_{p} & \text { if } \zeta \in \mathcal{F}_{y} \\
{\left[\begin{array}{l}
\hat{x}_{p}^{+} \\
x_{c}^{+}
\end{array}\right]} & =\left[\begin{array}{cr}
I & 0 \\
K_{p} & 0
\end{array}\right]\left[\begin{array}{l}
\hat{x}_{p} \\
x_{c}
\end{array}\right]+\left[\begin{array}{c}
0 \\
K_{y}
\end{array}\right] \eta & & \text { if } \zeta \in \mathcal{J}_{y} \\
u & =C_{\mathcal{H}} \bar{x}+D_{\mathcal{H}} y_{p} &
\end{array}
$$

and, interconnected to (2), it leads to the following hybrid closed-loop system

$$
\begin{array}{rlrl}
\dot{x} & =A x & & \text { if } \zeta \in \mathcal{F}_{y} \\
{\left[\begin{array}{c}
\hat{x}_{p}^{+} \\
x_{c}^{+} \\
e^{+}
\end{array}\right]} & =\left[\begin{array}{ccc}
I & 0 & 0 \\
K_{p} & 0 & K_{y} \bar{C}_{p} \\
0 & 0 & I
\end{array}\right]\left[\begin{array}{c}
\hat{x}_{p} \\
x_{c} \\
e
\end{array}\right] \quad \text { if } \zeta \in \mathcal{J}_{y} \\
y_{p} & =C x, & &
\end{array}
$$


where $A$ and $C$ are defined in (4). Moreover, the flow and jump sets are given by

$$
\begin{aligned}
& \mathcal{F}_{y}=\left\{\zeta: \zeta^{T} M \zeta \leq-\tilde{\alpha} \zeta^{T} \tilde{P} \zeta \text { or } \zeta^{T} \tilde{P} \zeta \leq \rho\right\} \\
& \mathcal{J}_{y}=\left\{\zeta: \zeta^{T} M \zeta \geq-\tilde{\alpha} \zeta^{T} \tilde{P} \zeta \text { and } \zeta^{T} \tilde{P} \zeta \geq \rho\right\}
\end{aligned}
$$

where

$$
\begin{gathered}
M=\operatorname{He}\left(\tilde{P}\left[\begin{array}{ccc}
A_{p} & B_{p} & -B_{p} K_{y} \\
B_{c} & A_{c} & 0 \\
0 & 0 & 0
\end{array}\right]\right), \\
\tilde{P}=\left[\begin{array}{ccc}
I & 0 & 0 \\
0 & I & -K_{y}
\end{array}\right]^{T} P\left[\begin{array}{ccc}
I & 0 & 0 \\
0 & I & -K_{y}
\end{array}\right],
\end{gathered}
$$

$P$ is given in Assumption 3, $0<\tilde{\alpha} \leq \alpha$ and the scalar $\rho>0$ is related to the practical stability and can be chosen arbitrarily small.

Theorem 2: Consider a plant-controller pair (2),(10) with the sets $\mathcal{F}_{y}$ and $\mathcal{J}_{y}$ in $(12), K_{p}=-P_{c}^{-1} P_{p c}^{T}$, satisfying Assumptions 1, 2 and 3 and with $A_{e}$ Hurwitz.

Then for any $K_{y} \in \mathbb{R}^{n_{c} \times q}$, the hybrid controller (10) guarantees that the set (9) is globally asymptotically stable for the closed-loop system (11), namely the origin is practically GAS ( $\rho$ can be arbitrarily small).

Remark 4: Note that the sets in (12) are defined based on the only quantities available for feedback, namely $\zeta=(\bar{x}, \eta)$. Similarly, the jump rule in (11) only requires knowledge of $\eta$ (and not the whole error $e$ ) because of the matrix $\bar{C}_{p}$ appearing in the $(2,3)$ entry of the jump matrix.

\section{Comments and remarks}

Remark 5: For the linear case addressed here, the state feedback techniques of [13], [14], induce exponential stability of the origin of the state space, whereas for the controllers (6) and (10) proposed here Theorems 1 and 2 only guarantee global practical asymptotic stability of the origin (see (9)), namely GAS of the set. The set $\mathcal{A}$ shrinks to the origin as the free parameter $\rho>0$ becomes arbitrarily small. The need for the set $\mathcal{A}$ arises from the fact that $\hat{x}_{p}^{+}=\hat{x}_{p}$, therefore the Luenberger observer is not affected by the resets. To guarantee the convergence to zero of $e$, we then need to avoid Zeno solutions. To this aim, the arbitrarily small neighborhood $\mathcal{A}$ of the origin is removed from the jump set and practical asymptotic stability is established. $\circ$

Remark 6: Note that Theorem 1 is a corollary of Theorem 2, in fact, if we select $K_{y}=0$ in (11), (12), then for all $(\bar{x}, e)$ the function $\tilde{V}(\zeta)=\zeta^{T} \tilde{P} \zeta$ is equal to $V(\bar{x})=\bar{x}^{T} P \bar{x}$ and moreover $\mathcal{F}_{y} \equiv\left(\mathcal{F} \times \mathbb{R}^{n_{p}}\right)$ and $\mathcal{J}_{y} \equiv\left(\mathcal{J} \times \mathbb{R}^{n_{p}}\right)$, that is, the two control techniques match. The same happens when the error estimation $e$ is zero. This means that (6) and (10) should have different behavior only during the observer transient. However, it is useful to keep the two solutions distinct because the implementation suggested in Section II$\mathrm{B}$ is simpler than the one of Section II-C.
Because of Remark 6, the two schemes have many common features. For this reason, in the sequel, we will present some common properties starting from $\tilde{V}$, because the corresponding property for $V$ may be obtained by selecting a $\tilde{V}$ with $K_{y}=0$ and by replacing $\zeta \in \mathcal{F}_{y}$ (respectively, $\zeta \in \mathcal{J}_{y}$ ) by $\bar{x} \in \mathcal{F}$ (respectively, $\bar{x} \in \mathcal{J}$ ).

As we are considering linear differential and difference equations, we can resort to a quadratic Lyapunov-like function $\zeta \mapsto \tilde{V}(\zeta)=\zeta^{T} \tilde{P} \zeta$ (respectively, $\bar{x} \mapsto V(\bar{x})=\bar{x}^{T} P \bar{x}$ ). This function has a crucial role for the supervisor block in the controller architecture of Fig. 1, to obtain that:

i. the function $\zeta \mapsto \tilde{V}(\zeta)$ (respectively, $\bar{x} \mapsto V(\bar{x})$ ) does not increase across the resets;

ii. some components of the function $\zeta \mapsto \tilde{V}(\zeta)$ (respectively, $\bar{x} \mapsto V(\bar{x}))$ do not increase across the flow;

iii. the hybrid system flows after each reset and, in particular, Zeno solutions never occur.

We can comment in more detail the three items above. First, note that the controller state $x_{c}$ is reset to the value

$$
\begin{aligned}
\arg \min _{x_{c}}\left[\begin{array}{c}
\hat{x}_{p} \\
x_{c} \\
\eta
\end{array}\right]^{T} \tilde{P}\left[\begin{array}{c}
\hat{x}_{p} \\
x_{c} \\
\eta
\end{array}\right] & =-P_{c}^{-1} P_{p c}^{T} \hat{x}_{p}+K_{y} \eta \\
& =: K_{p} \hat{x}_{p}+K_{y} \eta .
\end{aligned}
$$

In this way we achieve the non-increase of $\tilde{V}$ (respectively, $V)$ across resets stated in item (i). Indeed, the reset map minimizes $\tilde{V}$ (respectively, $V$ ) with respect to $\hat{x}_{p}$ and $\eta$ (respectively, with respect to $\hat{x}_{p}$ only).

Item (ii) is obtained by definition of the flow set. In particular, the first inequality requires that the components of the time-derivative of $\tilde{V}$ (respectively, $V$ ), not depending on $e$, be strictly decreasing. This explains why only when $e=0$ we may be sure that all the components of $\tilde{V}$ (respectively, $V$ ) are decreasing across the flow.

Finally, item (iii) on the avoidance of Zeno solutions is necessary to ensure that $e$ converge to zero and is achieved by combining two facts:

a. homogeneity of the system (due to the linearity of the equations),

b. the guarantee of a good flow condition, that is, if $\zeta \in \mathcal{J}_{y}$ (respectively, $\bar{x} \in \mathcal{J}$ ) then $\zeta^{+} \in \mathcal{F}_{y} \backslash \mathcal{J}_{y}$ (respectively, $\left.\bar{x}^{+} \in \mathcal{F} \backslash \mathcal{J}\right)$.

From homogeneity of the system, we deduce that after each jump the solution flows for at least an interval time $\tau_{\text {min }}$ before it reaches the boundary of the flow set. Such a $\tau_{\min }$ is a finite strictly positive scalar because this minimum is carried out over a compact set.

Item $b$. is guaranteed by the following claim.

Claim 1: Under the conditions of Theorem 1 (respectively, Theorem 2), if $\bar{x} \in \mathcal{J}$ (respectively, $\zeta \in \mathcal{J}_{y}$ ), then $x_{c} \neq K_{p} \hat{x}_{p}$ (respectively, $x_{c} \neq K_{p} \hat{x}_{p}+K_{y} \eta$ ).

The flow and jump sets in (7) are exactly like the ones in [13], except for the arbitrarily small neighborhood of the 
origin, $V(\bar{x}) \leq \rho$. However, we notice that the flow and jump sets here have a different meaning because they are expressed in the coordinates $\bar{x}$. The flow and jump sets in (12) and the Lyapunov-like function $\zeta \mapsto \tilde{V}(\zeta)$ exploit more information related to the output estimation error of the observer. Only when $e=0$ (that is, when $\hat{x}_{p}=x_{p}$ ) the Lyapunov-like function $\bar{x} \mapsto V(\bar{x})$ (respectively, $\zeta \mapsto \tilde{V}(\zeta)$ ) coincides with the function in [13], [14] More generally, when $e \neq 0$, we know that $e$ converges exponentially to zero and the properties of the designs in [13], [14] are asymptotically recovered for the output feedback case.

In Section III, we present data simulations and emphasize how to generate a function $\zeta \mapsto \tilde{V}(\zeta)$ (therefore also a function $\bar{x} \mapsto V(\bar{x}))$ satisfying the properties above.

\section{SIMULATIONS}

Following the technique porposed in [13, §4], let us consider the following Lyapunov-like function

$\tilde{V}(\zeta)=V_{p}\left(\hat{x}_{p}\right)+\left(x_{c}-K_{p} \hat{x}_{p}-K_{y} \eta\right)^{T} P_{c}\left(x_{c}-K_{p} \hat{x}_{p}-K_{y} \eta\right)$

with $V_{p}\left(\hat{x}_{p}\right)=\hat{x}_{p}^{T} \bar{P} \hat{x}_{p}$ and $\bar{P}$ symmetric and positive definite. Then, it is clear that for all $x$, any gain $K_{p}$ and $K_{y}$, the reset map $x_{c}=K_{p} \hat{x}_{p}+K_{y} \eta$ satisfies (13). Moreover, equation (14) written in matrix form becomes

$$
\begin{aligned}
\tilde{V}(\zeta) & =\left[\begin{array}{c}
\hat{x}_{p} \\
x_{c} \\
\eta
\end{array}\right]^{T}\left[\begin{array}{ccc}
\bar{P}_{p}+K_{p}^{T} P_{c} K_{p} & -K_{p}^{T} P_{c} & K_{p}^{T} P_{c} K_{y} \\
\star & P_{c} & -P_{c} K_{y} \\
\star & \star & K_{y}^{T} P_{c} K_{y}
\end{array}\right]\left[\begin{array}{c}
\hat{x}_{p} \\
x_{c} \\
\eta
\end{array}\right] \\
& =\zeta^{T} \tilde{P} \zeta:=\zeta^{T}\left[\begin{array}{c|c}
P & P_{p y} \\
& P_{c y} \\
\hdashline \star & P_{y}
\end{array}\right] \zeta,
\end{aligned}
$$

where $P$ was defined in Assumption 3.

Note that considering $K_{p}=-P_{c}^{-1} P_{p c}^{T}$, we have $P_{p}=$ $\bar{P}_{p}+K_{p}^{T} P_{c} K_{p}=\bar{P}_{p}+P_{p c} P_{c}^{-1} P_{p c}^{T}$ and then (5) can be equivalently rewritten as

$$
\operatorname{He}\left(\bar{P}_{p}\left(A_{p}+B_{p} K_{p}\right)\right)<-\alpha \bar{P}_{p} .
$$

At this point, if one selects $K_{p}$ as a stabilizer for the pair $\left(A_{p}, B_{p}\right)$, it is always possible to find a $\bar{P}_{p}=\bar{P}_{p}^{T}>0$ and $\alpha>0$ to satisfy (16) (or equivalently (5)). Once $K_{p}, \bar{P}_{p}$ and $\alpha$ are obtained, choosing any $K_{y}$ and $P_{c}$ symmetric large enough to make $P$ positive definite, we can construct $\tilde{P}$ from (15) (or equivalently from (12d)) and the flow and jump sets, $\mathcal{F}_{y}$ and $\mathcal{J}_{y}$. Note also that selecting $K_{y}=0$ we obtain $P, \mathcal{F}$ and $\mathcal{J}$.

To show the effectiveness of our result an historical simulation example is presented.

Consider now the plant $P(s)=\frac{s+1}{s(s+0.2)}$ introduced in [9] and discussed in [14] and [19]. According with (2), a possible realization is

$$
\left[\begin{array}{c|c}
\bar{A}_{p} & \bar{B}_{p} \\
\hline C_{p} & D_{p}
\end{array}\right]=\left[\begin{array}{cc|c}
-0.6 & 0.6 & -1 \\
-0.4 & 0.4 & 1 \\
\hline 0 & 1 & 0
\end{array}\right]
$$

TABLE I

HYBRID CONTROLLER SETTING PROPOSED

\begin{tabular}{|cc|c|c|}
\hline \multicolumn{2}{|c|}{$\bar{P}_{p}$} & $K_{p}^{T}$ & $\alpha$ \\
\hline 0.0097785 & -0.0096375 & 0.0594992 & $7.784 \cdot 10^{-6}$ \\
-0.0096375 & 0.99990 & -4.83065 & \\
\hline
\end{tabular}

Notice that the pair $\left(\bar{C}_{p}, \bar{A}_{p}\right)$ is observable. For a reasonable comparison, to design the continuous-time part (3a) of the controllers, we select the matrices $\bar{A}_{c}, \bar{B}_{c}, \bar{C}_{c}$ and $\bar{D}_{c}$ to define the same closed loop used in [9] and [14] (which is in negative feedback with a FORE), obtaining

$$
\left[\begin{array}{c|c}
\bar{A}_{c} & \bar{B}_{c} \\
\hline C_{c} & D_{c}
\end{array}\right]=\left[\begin{array}{c|c}
-1 & -1 \\
\hline 1 & 0
\end{array}\right],
$$

and the observer via an LQG synthesis that returns $L=$ $\left[\begin{array}{ll}0.26 & 1.37\end{array}\right]^{T}$. Finally, controller (11) is manually tuned with $K_{y}=-2$.

For the hybrid part of our controller, we exploit the optimal configuration presented for the static state feedback in [14] for the overshoot reduction, see Table I. As the basic idea of this optimization is to approximate the Lyapunov-like function, used to define the flow and jump sets, to the norm of the plant output (i.e. $\tilde{V}(\zeta) \approx y_{p}^{T} y_{p}$ (respectively, $\left.V(\bar{x}) \approx y_{p}^{T} y_{p}\right)$ ), we select $P_{c}=10^{-10}$ (ideally the smallest $P_{c}$ that satisfies all the conditions in Assumption 3) in such a way that the influence of the sub-state $x_{c}$ be reduced. Moreover we set $\tilde{\alpha}=10^{-8}$ to enlarge as much as possible the flow set and $\rho=10^{-3}$.

Fig. 2 depicts the input and output behavior of the hybrid systems (8) (dashed line) and (11) (dot-dashed line) compared to the linear case, to the technique in [9] and to the technique in [14].

All the controllers have zero initial conditions whereas the plant state starts from $x_{p}(0,0)=-\left[\begin{array}{ll}1 & 1\end{array}\right]^{T}$.

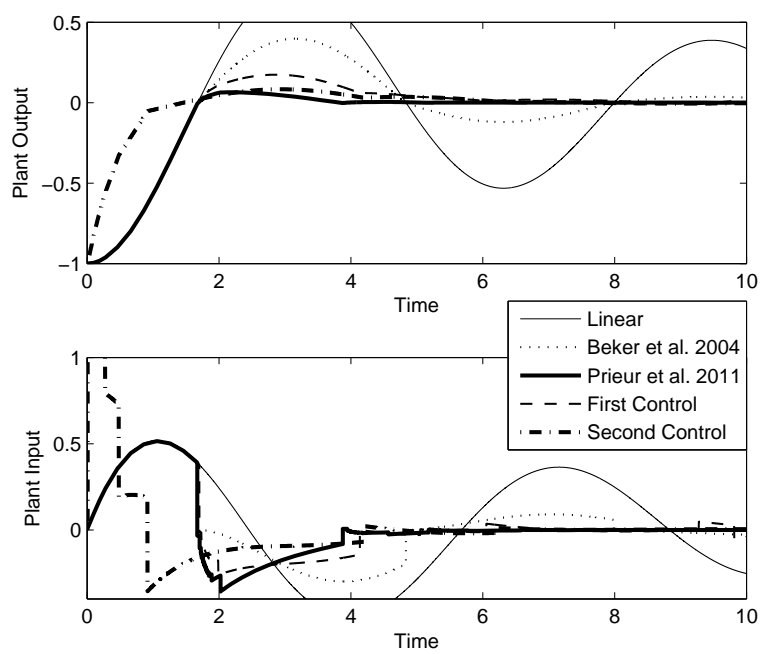

Fig. 2. Simulation results using the hybrid controllers (6) and (10), compared to the linear case, to the FORE in [9] and to the hybrid controller with optimal static state feedback given in [14]. 
As expected, the undesired effects of the dynamics of the observer affect the controller (6) exhibiting a larger overshoot than the static state feedback of [14] caused by the observer transient. The second control technique is capable to partially compensate for this gap recovering some performance for the output feedback case. Note that the faster rise time might misleadingly convey the idea that the dash-dotted response is more desirable than the bold one. This is not the case because we are assessing overshoot reduction here and the shorter rise time is obtained by the dash-dotted curve at the price of a larger control input (see the lower plot). We remark that, although the FORE in [9] does not introduce further dynamics for the hybrid closed-loop system, it exhibits a larger overshoot than (6) and (10).

The results in Fig. 2 are purely illustrative of the potential of the proposed controller architectures. Indeed the parameters of the example study are empirically selected. In particular, the problem of how to select $K_{y}$ is still open.

\section{CONCLUSIONS}

This paper proposes the use of hybrid loops to augment a (not necessarily stabilizing) linear continuous-time controller in output feedback with a linear continuous-time plant. The designed hybrid loops allow to guarantee stability of the arising hybrid closed-loop system. In particular, the output feedback case has been solved here by relying on a Luenberger observer providing an estimate of the plant state, which is used to define the flow and jump sets, and the jump law.

Future research directions include deeper investigations about performance of the method here proposed and seeking for design techniques aimed at inducting performance properties that would not be achievable with continuous-time only controllers.

\section{REFERENCES}

[1] R. Sanfelice, R. Goebel, and A. Teel, "Invariance Principles for Hybrid Systems With Connections to Detectability and Asymptotic Stability." IEEE Transactions on Automatic Control, vol. 52, no. 12, pp. 22822297, 2007.

[2] R. Goebel, R. Sanfelice, and A. Teel, "Hybrid Dynamical Systems." IEEE Control Systems Magazine, vol. 29, no. 2, pp. 28-93, April 2009.

[3] R. Goebel and A. Teel, "Solutions to Hybrid Inclusions via Set and Graphical Convergence With Stability Theory Applications." Automatica, vol. 42, no. 4, pp. 573-587, 2006.

[4] J. Hespanha and A. Morse, "Stabilization of Nonholonomic Integrators via Logic-based Switching." Automatica, vol. 35, no. 3, pp. 385-393, 1999.

[5] J. Hespanha, D. Liberzon, and A. Morse, "Hysteresis-based Switching Algorithms for Supervisory.” Automatica, vol. 39, no. 2, pp. 263-272, 2004.

[6] C. Prieur, "Asymptotic Controllability and Robust Asymptotic Stabilizability." SIAM Journal on Control and Optimization, vol. 43, no. 5, pp. 1888-1912, 2005.

[7] W. Aangenent, G. Witvoet, W. Heemels, M. van de Molengraft, and M. Steinbuch, "Performance Analysis of Reset Control Systems." International Journal of Robust and Nonlinear Control, vol. 20, no. 11, pp. 1213-1233, 2010.

[8] D. Nešić, A. Teel, and L. Zaccarian, "Stability and Performance of SISO Control Systems with First Order Reset Elements." IEEE Transactions on Automatic Control, pp. 563-568, 2011, in press.

[9] O. Beker, C. Hollot, and Y. Chait, "Fundamental Properties of Reset Control Systems.” Automatica, vol. 40, no. 6, pp. 905-915, 2004.
[10] F. Safei, J. Hespanha, and G. Stewart, "Quadratic Optimization for Controller Initialization in Multivariable Switching Systems." in Proceedings of the 2010 American Control Conference, Baltimore, Maryland, USA, June 2010, pp. 2511-2516.

[11] W. Haddad, V. Chellaboina, and N. Kablar, "Non-linear Impulsive Dynamical Systems. Part I: Stability and Dissipativity.” International Journal of Control, vol. 74, no. 17, pp. 1631-1658, 2001.

[12] — "Non-linear Impulsive Dynamical Systems. Part II: Stability of Feedback Interconnections and Optimality." International Journal of Control, vol. 74, no. 17, pp. 1659-1677, 2001.

[13] C. Prieur, S. Tarbouriech, and L. Zaccarian, "Guaranteed Stability for Nonlinear Systems by Means of a Hybrid Loop." in Proceedings of the 8th IFAC Symposium on Nonlinear Control Systems (NOLCOS), Bologna, Italy, September 2010, pp. 72-77.

[14] $\_$, "Improving the Performance of Linear Systems by adding a Hybrid Loop." in 18th IFAC World Congress, Milano, Italy, September 2011.

[15] F. Forni, S. Galeani, D. Nešić, and L. Zaccarian, "Lazy Sensors for the Scheduling of Measurements Samples Transmission in Linear Closed Loops Over Networks." in Proceedings of the 49th IEEE Conference on Decision and Control, Atlanta (GA), USA, December 2010, pp. 6469-6474.

[16] J. Aubin, J. Lygeros, M. Quincampoix, S. Sastry, and N. Seube, "Impulse Differential Inclusions: a Viability Approach to Hybrid Systems." IEEE Transactions on Automatic Control, vol. 47, no. 1, pp. 2-20, 2002.

[17] J. Lygeros, K. Johansson, S. Simić, J. Zhang, and S. Sastry, "Dynamical Properties of Hybrid Automata." IEEE Transactions on Automatic Control, vol. 48, no. 1, pp. 2-17, 2003.

[18] R. Goebel and A. Teel, "Lyapunov Characterization of Zeno Behavior in Hybrid Systems." in Proceedings of the 47th IEEE Conference on Decision and Control, Cancun, Mexico, December 2008, pp. 27522757.

[19] D. Nešić, L. Zaccarian, and A. Teel, "Stability Properties of Reset Systems." in Proceedings of the 16th IFAC World Congress, vol. 16, no. 1, 2005, pp. 67-72.

[20] F. Clarke, Y. Ledyaev, R. Stern, and P. Wolenski, Nonsmooth Analysis and Control Theory. New York: Springer-Verlag, 1998. 